\title{
Dictyostelium Cell Fixation: Two Simple Tricks
}

\author{
Michael Koonce ${ }^{1, *}$, Irina Tikhonenko ${ }^{1}$ and Ralph Gräf ${ }^{2}$ \\ 1 Division of Translational Medicine, Wadsworth Center, NYS Department of Health, Albany, NY 12237, USA; \\ irina.tikhonenko@health.ny.gov \\ 2 Department of Cell Biology, University of Potsdam, 14476 Potsdam-Golm, Germany; rgraef@uni-potsdam.de \\ * Correspondence: michael.koonce@health.ny.gov; Tel.: +1-518-486-1490
}

Received: 16 June 2020; Accepted: 1 July 2020; Published: 1 July 2020

\begin{abstract}
We share two simple modifications to enhance the fixation and imaging of relatively small, motile, and rounded model cells. These include cell centrifugation and the addition of trace amounts of glutaraldehyde to existing fixation methods. Though they need to be carefully considered in each context, they have been useful to our studies of the spatial relationships of the microtubule cytoskeletal system.
\end{abstract}

Keywords: Dictyostelium; cell fixation; microscopy; microtubule; cytoskeleton

\section{Introduction}

The striking mobility of Dictyostelium makes for an interesting and impactful model system to study dynamic cellular events [1]. Directed cell movement, rapid shape changes, and internal reorganization in response to extracellular cues are frequently accentuated here over more sedentary cell types and thus provide novel access to their study and understanding. However, one of the tradeoffs in a dynamic lifestyle is that these cells can be less tightly adherent to their substrate, and thus imaging and downstream structural analyses can present challenges that must be carefully considered.

For many years, we have tinkered with fixation and staining conditions in order to image cytoskeletal arrays and GFP-tagged proteins in D. discoideum [2-6]. Likely not alone, we have been challenged with difficulties in getting cells to sufficiently flatten to facilitate imaging and to remain attached to coverslips for downstream structural processing. In this brief report, we would like to share two insights that make a difference in our work, in the hopes that they benefit others as well.

\section{Results}

\subsection{Cell Flattening}

In the early 1980's, Fukui and colleagues developed an agar overlay procedure for D. discoideum that utilizes thin sheets of agarose to flatten cells and significantly improves some aspects of cellular imaging [7]. This is now a widely used method that gently restricts the experimental depth of field to around 1-3 $\mu \mathrm{m}$. Cells under agarose can undergo division and remain viable for hours in sealed chambers. This overlay remains our preferred method for live cell imaging, enabling us to follow individual microtubule (MT) motions in a minimal number of focal planes [8,9]. However, the procedure is a bit cumbersome to use for routine cell fixation, especially for multiple coverslips. The agar sheet takes some care to set up, only covers a small portion of the coverslip, and takes slightly longer for the fixative to penetrate to the cells, and cell loss (at least in our hands) can be significant upon sheet removal for downstream cell processing. With any perturbation, there are caveats to consider when flattening cells for long periods, and thus multiple strategies can be useful to distinguish between genuine and imposed phenotypes. 
As such an alternative, we have begun to spin cells directly onto coverslips. Five minutes at $400 \mathrm{~g}$ in a standard tabletop centrifuge not only promotes attachment but also cell spreading. A small volume of cell suspension is added to buffer overlying a coverslip in a centrifuge tube (Figure 1) and spun for five minutes. The coverslip is then lifted out and gently slid into a dish containing fixative. This approach eliminates the waiting period for cell settling; agarose is not required, and cells labeled with a tubulin antibody [10] appear very similar to those prepared by agar overlay (Figure 2).
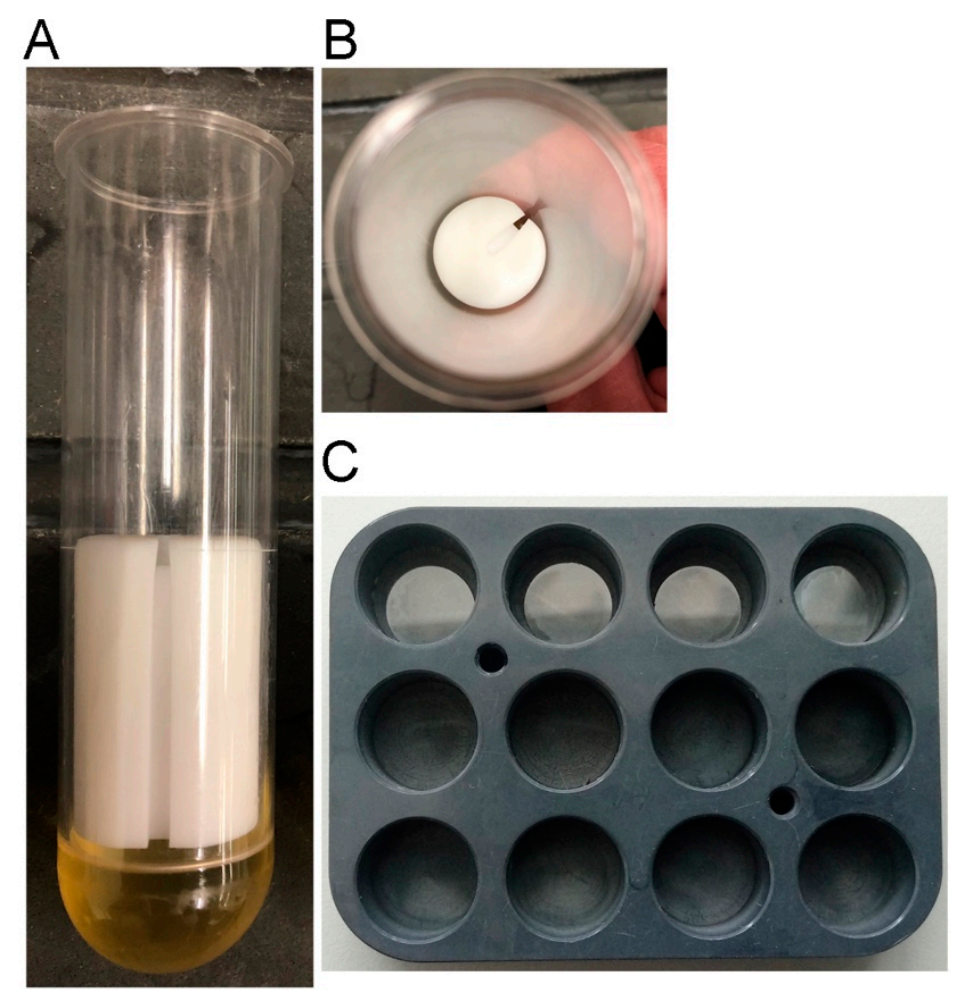

Figure 1. Centrifugation devices. Panel (A) shows a side view, panel (B) shows a top down view of a $50 \mathrm{~mL}$ Sorvall centrifugation tube fitted with an EPON base (yellow layer in A) and a white Delrin rod segment $(2.5 \mathrm{~cm}$ diameter, $3.75 \mathrm{~cm}$ length). The notch along the side is helpful to retrieve coverslips with forceps, allowing access to the underside of the coverslip for lifting. Panel (C) shows a modified multiwell holder for processing up to 12 samples at a time. The top row contains $12 \mathrm{~mm}$ round coverslips; these can be readily removed with curved forceps.

The take-home message here is that the organization of the MT array in centrifuged cells appears to be very similar if not identical to what we would select as the "best" cells from either direct fix or agar overlay procedures. However, a primary benefit is that they are significantly more plentiful on the centrifuged coverslip than in the other two preparations. For example, approximately $10 \%$ of the cells on a gravity settled coverslip are sufficiently flat enough to project a useful characterization of MT arrays for the types questions that interest us. On a centrifuged coverslip, this increases to at least $60 \%$ of the population. This percentage is similar in the agar overlay preparations, but centrifuged cells extend across the entire coverslip and do not suffer detachment due to agar sheet removal. 


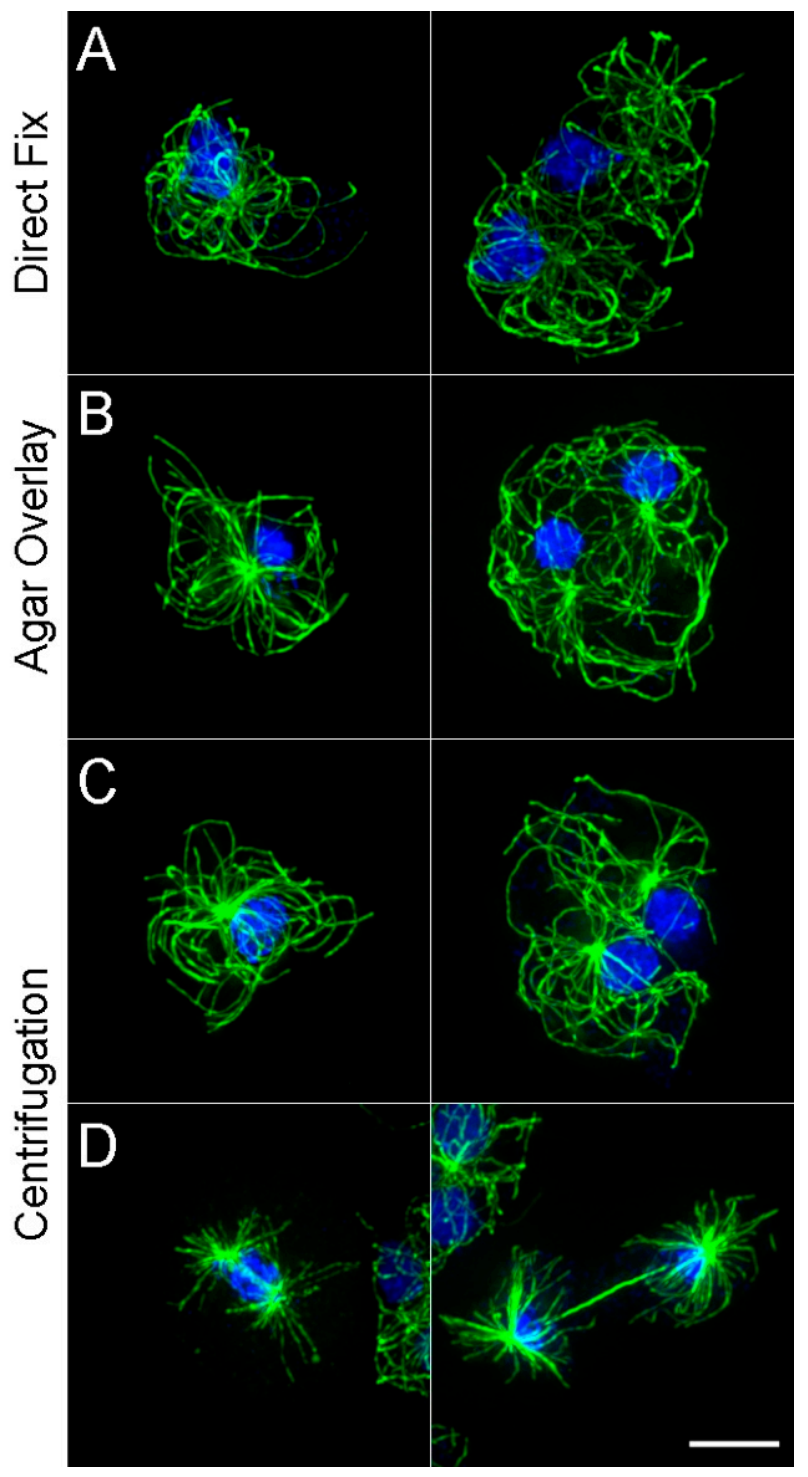

Figure 2. Examples of antibody-labeled microtube (MT) patterns in cells (A) directly added to coverslips and fixed after a $10 \mathrm{~min}$ period; (B) cells fixed after overlay with agar sheets; and (C,D) cells fixed after 5 min centrifugation onto coverslips. Panels (A-C) show interphase arrangements of mononucleated cells (left) and binucleates (right). Panel (D) shows mitotic cells in late anaphase (left) and late telophase (right). While the three different cell preparations look similar, it is important to note that there are typically far fewer flattened cells on the direct fix coverslips and substantially fewer cells in general on the agarose overlay coverslip. MTs are shown in green, DNA in blue, Scale bar $=5 \mu \mathrm{m}$.

\subsection{Cell Attachment}

The second perhaps underappreciated impact derives from a fixation recipe that includes a small amount of glutaraldehyde $(0.05 \%$ final). This concentration is not sufficient to preserve cell structure on its own, nor does it require autofluorescence quenching by sodium borohydride, but it substantially improves cell retention on the coverslip (Figure 3). Although formaldehyde alone or $-20^{\circ}$ methanol can provide structural preservation at the light microscopy (LM) level, cell loss from the coverslip is substantial at each wash or incubation step. This is particularly a problem for mitotic cells, since they are more rounded and less adherent than during interphase. The fixation recipe provided below does not interfere with GFP labels, and thus expressed tags can be imaged alongside antibody staining. Compared to the use of higher concentrations of glutaraldehyde (i.e., $0.5 \%$ ) as the sole fixative, 
this recipe also has the advantage that it improves preservation of MTs without the often-encountered negative effects that glutaraldehyde has on antigenicity for antibodies directed against other structures.

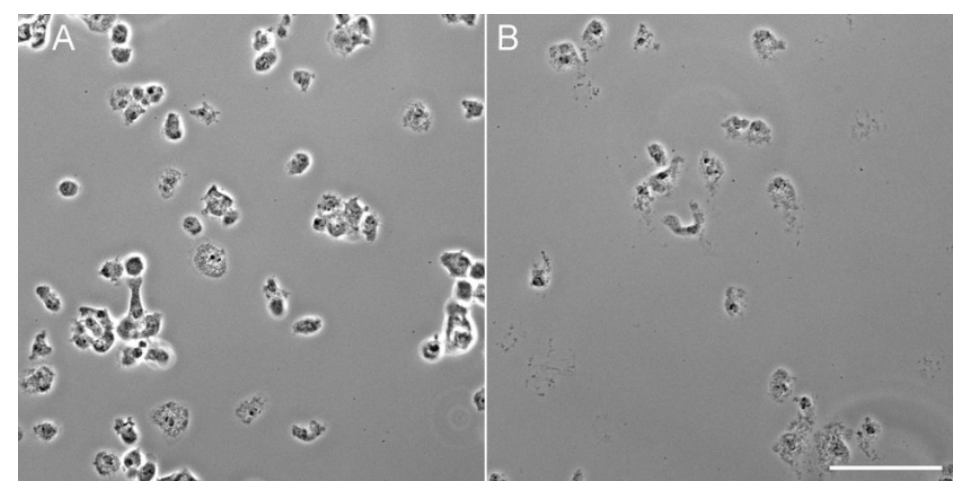

Figure 3. Phase contrast images of identical aliquots of $D$. discoideum cells spun onto coverslips and fixed (A) with or (B) without the addition of $0.05 \%$ glutaraldehyde and rinsed four times with buffer. Scale bar $=50 \mu \mathrm{m}$.

\section{Discussion}

As with any cell perturbation, centrifugation may not universally be a good idea and needs to be approached with caution. However, side-by-side comparisons of the MT array (interphase or mitotic cells) prepared by agar overlay or centrifugation are indistinguishable, and thus in our case, we feel the method affords a useful strategy for our imaging.

An important benefit is that this method can be applied to cells that do not readily attach to the coverslip. For example, treatment of $D$. discoideum with latrunculin A $(5 \mu \mathrm{M})$ results in actin filament disruption and cell rounding [11]. Centrifugation of Lat-A-treated cells onto coverslips followed by immediate fixation preserves a significant percentage of the population for downstream imaging. We have used this strategy to examine how the MT array may be integrated into the cortical actin meshwork.

Laboratories already comfortable with their staining procedures are not likely to do anything different, but for those frustrated that $D$. discoideum cells (or other cell types) are less adherent than mammalian tissue culture cells, these hints provide alternative strategies to consider.

\section{Experimental Design}

\subsection{Centrifugation}

For a limited number of samples, we used $50 \mathrm{~mL}$ polycarbonate Sorvall centrifuge tubes, which were roughly the same diameter as the ubiquitous $50 \mathrm{~mL}$ disposable screw cap tubes and could accommodate $18 \mathrm{~mm}^{2}$ coverslips. We partially filled a set with EPON resin (a common epoxy resin found in electron microscopy labs) to serve as a base, then inserted $3.75 \mathrm{~cm}$ long Delrin spacers of almost the same diameter as the centrifuge tube, for the coverslip to rest on. We added a small notch in the top of the spacer to facilitate forceps removal of the coverslip. In order for the spacer alone or multiple other configurations to work, one only needs a smooth surface that will support the coverslip; remain perpendicular to the centrifugal force; and, importantly, provide the ability to reach the coverslip with a pair of forceps for retrieval.

For processing more than a few coverslips, we used custom 12-(flat) well titer plates (about half the size of a standard 24-well plate) that fit into the buckets of a swing-out rotor. Round coverslips (12 mm) were placed into the wells prior to adding cells and centrifugation. There were also commercially available 24-well $0.17 \mathrm{~mm}$ glass bottom plates that fit into many swing-out buckets. Using these plates, the centrifugation method may more easily be applicable for live cell imaging. 
A clean coverslip was added to the tube followed by sufficient phosphate buffer to cover the coverslip by about $5 \mathrm{~mm}$. Using a micropipette, $\sim 100 \mu \mathrm{L}$ of $D$. discoideum cells were scraped from the bottom of a nearly confluent culture dish and gently added into the buffer volume directly over the coverslip. Depending on the desired cell density, this volume of cells can be adjusted. Tubes can be centrifuged as soon as convenient after adding cells; no settling period is required. While fixing D. discoideum cells directly in culture media should be avoided due to auto fluorescent components in some recipes, the dilution into buffer ( 1:50) and time over the brief spin period is sufficient to substantially reduce the fluorescent background.

The tubes or dishes were placed in a table top clinical centrifuge with a swinging bucket rotor and spun for five minutes ( $400 \mathrm{~g}$ setting). Coverslips were then quickly removed and placed cell side up in $35 \mathrm{~mm}$ petri dishes prefilled with fixative, and incubated for 15-30 min. All downstream washing and incubation steps were performed as usual for antibody or reporter labeling. The centrifugation time and force settings were not rigorously optimized; we tinkered with them, and these settings worked for our purpose.

\subsection{D. discoideum Fixative}

- $\quad 50 \%$ PHEM buffer (30 mM PIPES, 12.5 mM HEPES, pH 6.9, 4 mM EGTA, 1 mM MgCl 2 ). pH 6.9

- $3.7 \%$ formaldehyde

- $0.05 \%$ glutaraldehyde

- $0.1 \%$ Triton X-100

Coverslips are acid washed by soaking in $1 \mathrm{~N} \mathrm{HCl}$ for one hour, followed by rinsing with milli $\mathrm{Q}$ $\mathrm{H}_{2} \mathrm{O}$, and a brief $100 \%$ ETOH wash, then air dried in a glass petri dish.

\subsection{Phosphate Development Buffer}

- $20 \mathrm{mM} \mathrm{KCL}$

- $2.5 \mathrm{mM} \mathrm{MgCl}_{2} \cdot 6 \mathrm{H}_{2} \mathrm{O}$

- $27 \mathrm{mM} \mathrm{NaH} 2 \mathrm{PO} 4 \cdot \mathrm{H}_{2} \mathrm{O}$

- $24 \mathrm{mM} \mathrm{Na}_{2} \mathrm{HPO}_{4}$

- $\quad \mathrm{pH} 6.4$ (see reference [12] for detail)

\subsection{Light Microscopy}

Imaging was performed on a DeltaVision microscope workstation using the softWoRx 2.50 imaging package. Z-series stacks were collected at $0.5 \mu \mathrm{m}$ step intervals and deconvolved. Maximum intensity projections of the entire stack are presented here, performed using FIJI [13]. DNA is labeled with Hoechst 33342. The panels were assembled using Adobe Photoshop.

Author Contributions: Conceptualization, M.K., R.G.; methodology and investigation, M.K., I.T., and R.G.; resources, M.K., I.T., and R.G.; writing, M.K., R.G. All authors have read and agreed to the published version of the manuscript.

Funding: Our work is supported in part by the National Science Foundation (MCB-1510511 to M.P.K.).

Acknowledgments: We are grateful to Alexey Khodjakov for his long-term assistance with cellular imaging.

Conflicts of Interest: The authors declare no conflict of interest. The funders had no role in the design of the study; in the collection, analyses, or interpretation of data; in the writing of the manuscript; or in the decision to publish the results. 


\section{References}

1. Kessin, R.H. Dictyostelium: Evolution, Cell Biology, and the Development of Multicellularity; Cambridge University Press: Cambridge, UK, 2001; p. 294.

2. Koonce, M.P.; McIntosh, J.R. Identification and immunolocalization of cytoplasmic dynein in Dictyostelium. Cell Motil. Cytoskel. 1990, 15, 51-62. [CrossRef] [PubMed]

3. Koonce, M.P.; Samsó, M. Overexpression of cytoplasmic dynein's globular head causes a collapse of the interphase microtubule network in Dictyostelium. Mol. Biol. Cell 1996, 7, 935-948. [CrossRef]

4. Rehberg, M.; Gräf, R. Dictyostelium EB1 is a genuine centrosomal component required for proper spindle formation. Mol. Biol. Cell 2002, 13, 2301-2310. [CrossRef]

5. Samereier, M.; Baumann, O.; Meyer, I.; Gräf, R. Analysis of Dictyostelium TACC reveals differential interactions with CP224 and unusual dynamics of Dictyostelium microtubules. Cell. Mol. Life Sci. 2010, 68, $275-287$. [CrossRef]

6. Tikhonenko, I.; Magidson, V.; Gräf, R.; Khodjakov, A.; Koonce, M.P. A kinesin-mediated mechanism that couples centrosomes to nuclei. Cell. Mol. Life Sci. 2013, 70, 1285-1296. [CrossRef] [PubMed]

7. Fukui, Y.; Yumura, S.; Yumura, T.K. Agar-overlay immunofluorescence: High-resolution studies of cytoskeletal components and their changes during chemotaxis. Methods Cell Biol. 1987, 28, 347-356.

8. Samereier, M.; Meyer, I.; Koonce, M.P.; Gräf, R. Live cell-imaging techniques for analyses of microtubules in Dictyostelium. Methods Cell Biol. 2010, 97, 341-357.

9. Odell, J.; Sikirzhytski, V.; Tikhonenko, I.; Cobani, S.; Khodjakov, A.; Koonce, M. Force balances between interphase centrosomes as revealed by laser ablation. Mol. Biol. Cell 2019, 30, 1705-1715. [CrossRef]

10. Piperno, G.; Fuller, M.T. Monoclonal antibodies specific for an acetylated form of alpha-tubulin recognize the antigen in cilia and flagella from a variety of organisms. J. Cell Biol. 1985, 101, 2085. [CrossRef] [PubMed]

11. Gerisch, G.; Bretschneider, T.; Müller-Taubenberger, A.; Simmeth, E.; Ecke, M.; Diez, S.; Anderson, K. Mobile actin clusters and traveling waves in cells recovering from actin depolymerization. Biophys. J. 2004, 87, 3493-3503. [CrossRef]

12. Soll, D.R. Methods for manipulating and investigating developmental timing in Dictyostelium discoideum. Methods Cell Biol. 1987, 28, 413-431. [PubMed]

13. Schindelin, J.; Arganda-Carreras, I.; Frise, E.; Kaynig, V.; Longair, M.; Pietzsch, T.; Preibisch, S.; Rueden, C.; Saalfeld, S.; Schmid, B.; et al. FIJI: An open-source platform for biological-image analysis. Nat. Methods 2012, 9, 676. [CrossRef] [PubMed]

(C) 2020 by the authors. Licensee MDPI, Basel, Switzerland. This article is an open access article distributed under the terms and conditions of the Creative Commons Attribution (CC BY) license (http://creativecommons.org/licenses/by/4.0/). 